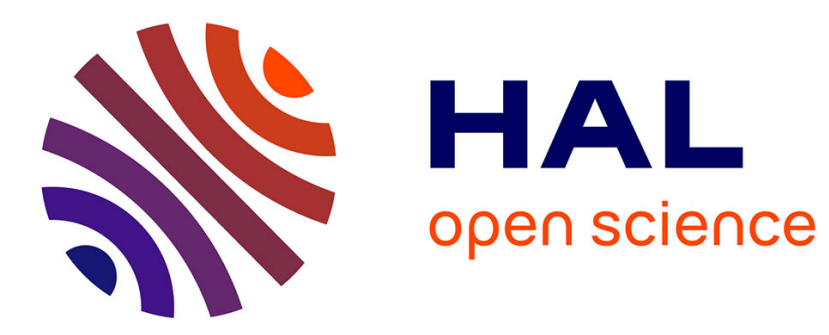

\title{
Le transfert et le temps
}

Alain Vanier

\section{- To cite this version:}

Alain Vanier. Le transfert et le temps. Topique - Revue freudienne, 2009, Ethique et technique de la psychanalyse, 106, pp.149-155. 10.3917/top.106.0149 . hal-01524061

\section{HAL Id: hal-01524061 \\ https://hal.science/hal-01524061}

Submitted on 16 Aug 2017

HAL is a multi-disciplinary open access archive for the deposit and dissemination of scientific research documents, whether they are published or not. The documents may come from teaching and research institutions in France or abroad, or from public or private research centers.
L'archive ouverte pluridisciplinaire HAL, est destinée au dépôt et à la diffusion de documents scientifiques de niveau recherche, publiés ou non, émanant des établissements d'enseignement et de recherche français ou étrangers, des laboratoires publics ou privés. 


\section{Le transfert et le temps ${ }^{1}$}

Alain Vanier

«Sur la clinique, le transfert et le temps »²a été l'occasion de ma rencontre avec l'œuvre de François Perrier. Il m'est apparu comme un véritable Ferenczi lacanien, peut-être par le lien si compliqué qu'il entretînt avec son analyste, Lacan, mais surtout par son souci, non seulement de la clinique, mais plus encore de la chose analytique et de la position du psychanalyste dans le traitement. Il ne basculera pas dans les dérives de Ferenczi, qui essuyait les plâtres de cette époque de pionniers. Néanmoins cette période fut aussi première, puisqu'elle est celle du formidable essor de la psychanalyse en France.

Je me suis intéressé à cet article, il y a un peu plus d'une quinzaine d'années, à l'occasion d'un travail sur le temps en psychanalyse. Aujourd'hui, je propose de revenir sur ce texte en soulignant qu'il est aussi un commentaire de l'enseignement de Lacan, ou plutôt une réappropriation de notions lacaniennes que traitait le séminaire à cette époque. Il commente Lacan, le reprend, mais c'est, à proprement parler, du François Perrier au meilleur sens du terme. Plusieurs propositions de ce texte passeront pour des évidences aujourd'hui, mais elles n'avaient rien d'évident à l'époque. Le risque avec ces considérations devenues si évidentes, donnant lieu à un accord quasi général, est d'en faire des lieux communs. C'est pourquoi il n'est peut-être pas inutile de les revivifier.

Cet article de 1968 souligne une double fonction du temps, irréversibilité et réversibilité, ou plutôt irréversibilité et régression. La position du psychana-

1. Communication à la Journée scientifique de l'AIHP « François Perrier, psychanalyste », le 31 mai 2008.

2. F. Perrier (1968), « Sur la clinique, le transfert et le temps », La Chaussée d'Antin, t. 2, Paris, Albin Michel, 2008.

Topique, 2009, 106, 149-155. 
lyste par rapport au temps, et le traitement de la question du temps dans la cure est le discriminant qui spécifie la psychanalyse. C'est la première grande thèse de l'article, et cette spécificité relève du psychanalyste. Ainsi peut s'entendre la formule singulière de Lacan définissant la psychanalyse comme le traitement qu'on attend d'un psychanalyste.

Le titre de l'article est son plan. Il s'ouvre sur le récit d'une erreur de diagnostic : un psychotique adressé par Perrier à Laplanche avec un diagnostic de névrose obsessionnelle. Mais Perrier ne réduit pas cet événement à une simple erreur de diagnostic. Il s'agit pour lui d'un «cas double» et non d'un « cas limite». Il ouvre la question du statut du diagnostic et de sa fonction, et, dans ce cas, la différence entre le premier praticien en position de diagnostiquer, qui apprécie une névrose obsessionnelle et le second, en position de psychanalyste quidéclenche la psychose, effet du transfert. En effet, l'autre thème, c'est l'athéisme freudien comme déclencheur de la psychose, c'est-à-dire le rapport du psychanalyste au réel. Il ouvre aussi le problème temporel de la névrose à la psychose - Freud disait qu'une névrose obsessionnelle peut se compliquer en paranoïa. Cettequestion de l'irréversibilité de la structure sera rouverte par le dernier Lacan. Perrier utilise ici la notion de «réponse du réel». Le diagnostic est irrattrapable car il est lié à la position subjective du psychanalyste, «le premier clinicien n'ayant été que le promoteur d'un diagnostic et d'une indication thérapeutique, le patient ne pourrait plus lui revenir comme tel. En tant que sujet sur un divan référé à un analyste en situation, il n'était plus récupérable pour une vérification, parce que non conforme à celui qui nous avait fourni les coordonnées d'un examen initial. Nous-mêmes, alertés par notre collègue, n'étions plus le même que celui qui lisait des structures obsessionnelles, là où il y avait une structure psychotique qui n'avait pas encore trouvé l'occasion de faire sa preuve. $»^{3}$

Perrier interroge la clinique en jouant de 1'opposition diachronie/synchronie. Pour la médecine, il se réfère à Foucault et au sujet mort de la méthode anatomoclinique, au corps-cadavre de Bichat. Le médecin peut s'intéresser à la « diachronie des troubles », le diagnostic est toujours un «flash». Il ne s'agit pas de la synchronie sur fond de diachronie qui caractérise la position du psychanalyste. Mais pour la psychiatrie, le sujetn'est pas le sujet mort de la médecine, car « la différence avec l'axe médical - et elle est d'importance - réside en ceci, que, loin d'en passer par la mort, certitude anticipée du mot de la fin qui met en facteur le syndrome comme condition d'un savoir sur la maladie, le psychiatre se garde le fou ou le névrosé comme vivant. Au besoin, il s'interne avec lui dans un espace asilaire pour célébrer la permanence de la confrontation entre la médecine mentale et les énigmes de la folie. Ici, la situation mise à l'abri est mise en conserve. La situation est faite pour durer car le psychiatre est en présence d'une façon de vivre et non de mourir. » Pourtant le psychanalyste doit 
être un psychiatre, avoirune formation psychiatrique et connaître les grilles diagnostiques. Il doit être, ajoute Perrier citant Canguilhem, « un spécialiste de l'inter-régionalité des disciplines ». Ce qui, pourtant, ne suffit pas à spécifier sa pratique. En psychanalyse il y a l'inconscient et le psychanalyste doit être attentif à ce que la théorie psychanalytique ne fonctionne pas comme une grille supplémentaire, commeunepsychanalyse appliquée, quitrouverait saplacedans le concert des théories. En effet, «le destin de la cure dépendra de l'aptitude de son théoricien d'analyste à ne plus se solidariser lui-même avec sa propre théorie de l'inconscient, si telle est la sienne ». La psychanalyse est une méthode qui implique autant l'analyste que l'analysant. Elle est définie par une règle «qui est le propre d'une démarche clinique assumée par un freudien : à savoir qu'il s'y implique autrement que tout autre clinicien de par la règle à laquelle il se soumet, une fois qu'il l'a édictée pour le patient.

C'est cette règle qui renverse les données de l'acte clinique, défini comme durée de l'enquête au service du flash de la représentation diagnostique, dans ses multiples modèles nosologiques ou métapsychologiques. Dans l'analyse comme praxis, la représentation est au service du déchiffrage, la synchronie au service de la diachronie, et cela parce qu'un opérateur se définit comme l'axe de temporalisation de la question de l'autre, sans terme prévisible pour aucun des deux interlocuteurs ; et ce à partir d'un rythme programmé de rencontres valable pour les deux. Une conséquence s'ensuit [...], c'est le transfert. »

Le terme de clinique n'est donc pas approprié. La psychanalyse est au-delà, elle est un dépassement, voire un renversement de la clinique. Elle met l'accent sur la diachronie du discours et sur les mouvements temporels de la cure, en premier lieu l'après-coup. C'est pourquoi le psychanalyste est à la fois quelconque et irremplaçable, quelconque par sa formation, il occupe une fonction, il est un opérateur dans la cure ; il est irremplaçable, une fois la cure engagée, car son style, pourrions-nous dire, engage la dimension du transfert. En ce sens, deux cures sont incomparables. Et le principe du transfert tient à ce qu'un «opérateur se définit comme axe de temporalisation de la question de l'autre », ce qui est à entendre comme mouvement de la cure, direction pour reprendre le mot de Lacan, en rappelant que la régression est son orientation. Ce mouvement est à comprendre comme une « progression vers l'enfance »; non pas faire un adulte, ce qui est la position de la psychothérapie, mais rendre « le sujet apte à l'enfance».

Pourquoi le transfert est-il cette actualisation? Le psychanalyste incarne ce reste non historisé au-delà de sa dimension imaginaire, au-delà de cette relation à l'autre, au-delà de sa dimension symbolique au sens de l'alternance absence-présence. Il est l'axe de temporalisation de ce réel non-inscrit dans le temps. L'enjeu de l'analyse est ainsi d'inscrire dans le temps ce qui ne l'a pas été. François Perrier met l'accent sur la notion d'objet $a$, ce qui n'est pas si fréquent à l'époque chez les élèves de Lacan. Il est remarquable, tout ce texte en 
témoigne, que Perrier soit à ce point dans le temps des élaborations de Lacan, dans le temps de cet accent mis sur l'objet a comme réel. Cette approche conduit à une relecture de la répétition.

Au cœur du transfert, ce sont des fragments de temps passé qui se déplacent intemporellement dans le temps, dans la méconnaissance du présent », comme a pu l'écrire joliment Jacques Sédat ${ }^{4}$. Perrier relit deux textes sur le transfert de Lacan. En 1948 Lacan rappelle, que le «nœud inaugural du drame analytique $»^{5}$ est le transfert négatif, en tant qu'il renvoie au fondement du registre imaginaire. C'est-à-dire à la projection sur l'analyste « d'une des images plus ou moins archaïques qui, par un effet de subduction symbolique, dégradent, dérivent ou inhibent le cycle de telles conduites qui, par un accident de refoulement, a exclu du contrôle du moi telle fonction et tel segment corporel, qui par une action d'identification a donné sa forme à telle instance de la personnalité ». En 1951, Lacan donne cette autre définition : «Le transfert n'est rien de réel dans le sujet, sinon l'apparition, dans un moment de stagnation de la dialectique analytique, des modes permanents selon lesquels il constitue ses objets. ${ }^{6}$ Mais l'analyste, dans toute cure, vient incarner cette place fixe de la répétition, ce qui revient toujours à la même place, ce qui le situe aussi sur le versant du réel. Il est le dépôt de la face mortifère de la répétition, et, en même temps, il est le lieu à partir duquel peut se déployer ce qu'est le transfert. Ainsi «le transfert est une relation essentiellement liée au temps et à son maniement $»^{7}$. C'est pourquoi il est intéressant d'insister, non pas sur le temps de la séance ou pas seulement - vaste débat -, mais aussi sur leur rythme, leur régularité etc., ce dont les psychanalystes parlent peu. En effet, « le psychanalyste s'édicte à l'autre comme une instance répétitive, et [...] il a l'air de trouver ça tout naturel». Et Perrier ajoute : «Voire, on discutera du temps de la séance comme constante ou variable, selon la structure clinique en analyse, et le temps pour comprendre (qui est sûrement différent d'un praticien à l'autre). On fera la théorie de la rencontre, de la ponctuation et de la coupure. Mais on laissera, à notre connaissance, de côté, l'aptitude de l'analyste à s'assumer comme représentant de la répétition, et de la multiplication des répétitions au profit du plus grand nombre. Aptitude, servitude, passion, éthique ou assujettissement à un automatisme ?» Et encore : «Ce à quoi il [le patient] tient, dans le transfert et pour le transfert, c'est que l'analyste en tant que sujet vivant, et supposé désirant, et supposé libre vis-à-vis de tout contrôle hiérarchique qui le subordonnerait à quelque instance autoritaire, se voue de lui-même à cette fidélité d'horloge vivante du temps de l'autre. ${ }^{8}$ Pourtant, la répétition fait

4. J. Sédat, «La rencontre : trouvaille ou retrouvaille», in Revue Adolescence, $\mathrm{n}^{\circ} 26,2008$.

5. J. Lacan, «L'agressivité en psychanalyse» (1948), Écrits, Paris, Seuil, 1966.

6. J. Lacan, « Intervention sur le transfert» (1951), Écrits, op. cit.

7. J. Lacan, « Position de l'inconscient au Congrès de Bonneval, reprise de 1960 en 1964 », Écrits, op. cit.

8. F. Perrier (1968), « Sur la clinique, le transfert et le temps », op. cit. p. 222-223. 
partie du dispositif, ne serait-ce qu'à partir «d'un rythme programmé de rencontres ». En effet, le psychanalyste est «le répétiteur payé».

Lacan n'identifie pas le transfert à la répétition. Il utilisera la distinction aristotélicienne entre tuché et automaton pour préciser cet écart. Le transfert ne se réduit pas à la répétition, il est aussi invention, précisément parce que le sujet s'adresse à un analyste.

Peut-on dire que le psychanalyste n'a pas ce que l'autre désire en lui, et qu'il ne donne finalement que le «rien » de l'objet manquant? Il «donne son temps », et quelque prix qu'il le fasse payer, le « time is money » ne peut se retourner véridiquement en un «money is time». Ce qui donne toute sa valeur à la règle qui institue le paiement des séances manquées : «La dette symbolique réelle renvoie à celui qui s'instaure, au regard de l'intemporalité de l'inconscient, comme un variant d'intégration dans les scansion répétées de son rythme de permanence à l'autre. Et, jusqu'à plus amples informés, nous considérons que toutes les variantes de la cure, par rapport à quelque cure type que l'on voudra, ne peuvent toucher au principe de fixité dans la répétition qui fait du praticien l'astronome des éphémérides transférentielles, et tout autant l'étoile fixe des configurations signifiantes mises en orbite par la règle analytique. » $\mathrm{C}$ 'est «la condition sine qua non du transfert au sens spécifique du terme ». On peut situer cette dimension du manque du côté du temps, et l'associer à la question de l'objet $a$.

On donne donc du temps, ce rien qui n'est pas rien, ce temps qu'on ne possède pas. La référence au réel, à l'objet $a$ de Lacan, a une conséquence. Le psychanalyste n'est pas seulement sujet supposé savoir mais sujet supposé synchrone à la vérité de l'autre, ce qui fait du temps pour comprendre de l'analyste, sa résistance.

La synchronie c'est du temps hors temps, et le psychanalyste est hors le temps, comme a pu l'écrire Pierre Fédida. Tout le problème du temps pour la psychanalyse est dans cette discordance fondamentale dont témoigne le trauma qui, dès la période pré-psychanalytique, est présenté en deux temps, qui fait son temps inassignable. Il advient toujours trop tôt ou trop tard. Le trauma, c'est-à-dire le sexuel, trouve sa forme paradigmatique dans l'instauration diphasique de la sexualité, dimension humaine fondamentale pour Freud. Car ce que Lacan indique dans le sexuel freudien, c'est le réel. Sur un autre versant, un linguiste, Gustave Guillaume, a montré comment la pensée du temps et sa représentation ne peuvent jamais coïncider ${ }^{9}$. Son étude manifeste cette discordance du langage au plus intime de son fonctionnement. Nous nous représentons toujours le temps de façon spatiale - ainsi les schémas freudiens. Il y aurait matière à reprendre le débat entre Lacan et Derrida sur la primauté de la parole ou de l'écriture. Le primat du signifiant est primat de la temporalité qui fait de l'espace un mode

9. Gustave Guillaume, Temps et verbe, Honoré Champion, 1929, réédition 1984. 
de représentation - cette succession, cette précession du signifiant sur la lettre est logique, mais non chronologique. Elle nous intéresse car cette inscription spatiale a son lieu, le corps, où elle a une fonction de découpe. Par ce biais, aujourd'hui, toute une clinique s'attrape préférentiellement. Cette représentation, pour Gustave Guillaume, occulte le «temps opératif », qui est «le temps que l'esprit emploie pour réaliser » cette représentation imaginaire du temps. Il y a donc un décalage irréductible, formulé par Giorgio Agamben comme l'écart «entre le temps que nous sommes et le temps dans lequel nous sommes». Cet écart est, pour nous, celui du battement entre les deux signifiants qui assurent la représentation du sujet. Cet écart manifeste une autre figure du présent, non recouverte par la jointure du passé et de l'avenir, dans ces moments où l'homme n'est fugacement plus «l'être des lointains», dont l'instant est la figure réelle comme coupure, comme «présent qui n'est pas un passage», mais qui « se tient immobile sur le seuil du temps »(W. Benjamin). La matière temporelle n'est pas que langage, elle ne fait temps du langage que par une soustraction fondamentale qui ne s'inscrit pas dans le temps. Cette opération n'est pas sans reste. Ce reste, c'est l'objet de la psychanalyse, il est le temps même et notre division. Il manifeste une défaillance originaire du symbolique qui est le biais par lequel nous l'incorporons. En effet, il y a quelque chose de l'origine qui ne parvient pas à être premier, mais qui en témoigne toujours au présent, et fait retour éternellement. Dans la reprise du deuxième temps du trauma, tout comme dans la conception des stades, il y a toujours un reste qui excède ce retour, ou qui fait de ce retour un excès, qui fait qu'il n'est pas seulement retour mais présence, il est là. «L'origine, bien qu'étant une catégorie tout à fait historique, n'a pourtant rien à voir avec la genèse des choses. L'origine ne désigne pas le devenir de ce qui est né, mais bien ce qui est en train de naître dans le devenir et le déclin. ${ }^{10}$ Origine non primaire elle est cette part retranchée que le langage ne cesse de traiter ${ }^{11}$, elle est cette défaillance initiale au cœur du transfert, sa face cachée. Cet écart est ce que vise la cure qui cherche «sur fond de désespoir», écrit Perrier, à ouvrir «le désir à sa cause», ce qu'il appelle l'éthique freudienne.

Alain VANIER

14 bis, rue Raynouard

75016 Paris

10. W. Benjamin (1928), Origine du drame baroque allemand, trad. S. Muller, Paris, Flammarion, 1985.

11. Cf. A. Vanier, «Ce qui ne cesse pas de ne pas commencer», Cliniques Méditerranéennes, 
Alain Vanier - Le transfert et le temps

Résumé : La position du psychanalyste au regard du temps définit celle qu'il occupe dans le transfert. Telle est la thèse d'un article de François Perrier qui est ici commenté.

Mots-clés :Transfert - Temps - Clinique - François Perrier - Lacan.

Alain Vanier - Transference and Time

Summary : The position of the psychoanalyst in relation to time defines the position the analyst holds in transference. This is the main tenet of an article by François Perrier which this article in turn explores.

Key-words : Transference - Time - Clinical Practice - François Perrier - Lacan. 